\title{
Correction to: Prevalence and risk factors for hypoparathyroidism following total thyroidectomy in Spain: a multicentric and nation- wide retrospective analysis
}

\begin{abstract}
Juan J. Díez $\mathbb{D}^{1,20} \cdot$ Emma Anda $^{2} \cdot$ Julia Sastre $^{3} \cdot$ Begoña Pérez Corral ${ }^{4}$ - Cristina Álvarez-Escolá ${ }^{5}$ Laura Manjón ${ }^{6}$. Miguel Paja ${ }^{7}$. Marcel Sambo ${ }^{8} \cdot$ Piedad Santiago Fernández $^{9} \cdot$ Concepción Blanco Carrera $^{10} \cdot$ Juan Carlos Galofré ${ }^{11}$. Elena Navarro ${ }^{12}$ - Carles Zafón ${ }^{13} \cdot$ Eva Sanz $^{13} \cdot$ Amelia Oleaga $^{7}$ - Orosia Bandrés ${ }^{14} \cdot$ Sergio Donnay $^{15} \cdot$ Ana Megía $^{16}$. María Picallo ${ }^{8}$. Cecilia Sánchez Ragnarsson ${ }^{6}$. Gloria Baena-Nieto ${ }^{17}$ - José Carlos Fernández García ${ }^{18}$. Beatriz Lecumberri $^{5}$ - Manel Sahún de la Vega ${ }^{19} \cdot$ Ana R. Romero-Lluch ${ }^{12} \cdot$ Pedro Iglesias $^{1,20}$
\end{abstract}

Published online: 22 October 2019

(c) Springer Science+Business Media, LLC, part of Springer Nature 2019

\section{Correction to: Endocrine}

https://doi.org/10.1007/s12020-019-02014-8

The original version of the article contained a mistake in the given and family name of the author Juan Carlos Galofré.

Juan J. Díez

juanjose.diez@salud.madrid.org

1 Department of Endocrinology, Hospital Universitario Ramón y Cajal, Madrid, Spain

2 Department of Endocrinology, Complejo Hospitalario de Navarra, Pamplona, Spain

3 Department of Endocrinology, Complejo Hospitalario de Toledo, Toledo, Spain

4 Department of Endocrinology, Complejo Asistencial Universitario de León, León, Spain

5 Department of Endocrinology, Hospital Universitario La Paz, Madrid, Spain

6 Department of Endocrinology, Hospital Universitario Central de Asturias, Oviedo, Spain

7 Department of Endocrinology, Hospital Universitario de Basurto, Bilbao, Spain

8 Department of Endocrinology, Hospital Universitario Gregorio Marañón, Madrid, Spain

9 Department of Endocrinology, Complejo Hospitalario de Jaén, Jaén, Spain

10 Department of Endocrinology, Hospital Universitario Príncipe de Asturias, Alcalá de Henares, Madrid, Spain
The correct given and family name is given below:

Given Name: Juan Middle Name: Carlos Family Name: Galofré The original article has been corrected.
11 Department of Endocrinology, Clínica Universidad de Navarra, Pamplona, Spain

12 Department of Endocrinology, Hospital Universitario Virgen del Rocío, Sevilla, Spain

13 Department of Endocrinology, Hospital Universitari Vall d'Hebron, Barcelona, Spain

14 Department of Endocrinology, Hospital Royo Villanova, Zaragoza, Spain

15 Department of Endocrinology, Fundación Hospital de Alcorcón, Alcorcón, Madrid, Spain

16 Department of Endocrinology, Hospital Universitario de Tarragona Joan XXIII, IISPV, Universitat Rovira i Virgili, Ciberdem, Tarragona, Spain

17 Department of Endocrinology, Hospital de Jerez, Jerez de la Frontera, Cádiz, Spain

18 Department of Endocrinology, Hospital Virgen de la Victoria, Málaga, Spain

19 Department of Endocrinology, Hospital Moisès Broggi, Sant Joan Despí, Barcelona, Spain

20 Present address: Department of Endocrinology, Hospital Universitario Puerta de Hierro Majadahonda, Madrid, Spain 